\title{
Histórico e Fatores Associados à Criminalidade e Violência entre Dependentes de Crack
}

\author{
Bianca Aparecida Ribeiro Singulane - Universidade Federal de Juiz de Fora, Juiz de Fora, Brasil \\ Nayara Baptista Silva - Universidade Federal de Juiz de Fora, Juiz de Fora, Brasil \\ Laisa Marcorela Andreoli Sartes - Universidade Federal de Juiz de Fora, Juiz de Fora, Brasil
}

\begin{abstract}
Resumo
Este estudo buscou avaliar o histórico, envolvimento recente e fatores associados a violência e criminalidade entre dependentes de crack em tratamento em Comunidades Terapêuticas (CTs). Foram entrevistados 72 indivíduos, maiores de 18 anos, que estavam em tratamento em CTs do interior de Minas Gerais. Os participantes responderam ao Addiction Severity Index versão 6 (ASI6), Teste de Triagem do Envolvimento com Álcool, Cigarro e Outras Substâncias (ASSIST) e questionário sociodemográfico. Os resultados mostraram que a maioria tinha histórico de detenções e esteve recentemente envolvido com violência e atividades ilegais, como roubo, furto, tráfico de drogas e agressões. A regressão de Poisson mostrou que o maior nível de instrução teve um efeito protetor, enquanto problemas com emprego, psiquiátricos e prisão na adolescência estavam associados a crimes nos últimos seis meses. Um programa de reinserção ocupacional e educacional durante o tratamento e após a alta das CTs pode ser uma abordagem protetora para crimes.

Palavras-chave: crime, violência, crack, comunidade terapêutica
\end{abstract}

History and Factors Associated with Crime and Violence among Crack Addicts

\begin{abstract}
This study sought to describe the involvement of crack addicts in treatment in therapeutic communities with violence and crime and to identify associated factors. We interviewed 72 individuals, over 18 years old, who were treated in therapeutic communities of a midsize city of Minas Gerais. We used the instruments: Addiction Severity Index version 6 (ASI 6), The Alcohol, Smoking and Substance Involvement Screening Test (ASSIST) and a sociodemographic questionnaire. The results showed that most of them had a history of arrests and had recently been involved with violence and illegal activities such as robbery, theft, drug trafficking and aggression. Poisson regression analysis showed that the higher level of education appears as protection, while employment and psychiatric problems, and prison in adolescence were associated with crimes in the past six months. An occupational and educational reinsertion program during treatment and after discharge of CTs could be a protective approach to crimes.

Keywords: crime, violence, crack, therapeutic community
\end{abstract}

Historia y Factores Relacionados a la Delincuencia y Violencia entre Adictos de Crack

\begin{abstract}
Resumen
Este estudio tuvo como objetivo evaluar la historia, participación reciente y los factores asociados a la violencia y delincuencia entre adictos de crack tratados en comunidades terapéuticas (CTs). Fueron entrevistados 72 individuos, mayores de 18 años, que estaban en tratamiento en CTs del interior de Minas Gerais. Los participantes respondieron al Addiction Severity Index versión 6 (ASI6), Test para detectar adicción con alcohol, cigarrillos y otras sustancias (ASSIST) y un cuestionario sociodemográfico. Los resultados mostraron que la mayoría tenía histórico de detenciones y había estado recientemente envuelto con violencia y actividades ilegales tales como hurto, robo, tráfico de drogas y agresiones. El análisis de regresión de Poisson mostró que el nivel más alto de instrucción tuvo un efecto protector, mientras que problemas con el empleo, problemas psiquiátricos y prisión en la adolescencia estaban asociados a crímenes en los últimos seis meses. Un programa de reinserción laboral y educacional durante el tratamiento y después del alta de las CTs puede ser un abordaje de protección para los crímenes.

Palabras clave: crimen, violencia, crack, comunidad terapéutica
\end{abstract}

O consumo de rrack tem sido alvo de preocupação da comunidade acadêmica, profissionais de saúde e da sociedade, salientando-se como um problema de saúde pública (Cunha, Nicastri, Gomes, Moino, \& Peluso, 2004). Um levantamento recente realizado nas 26 capitais brasileiras e Distrito Federal acerca do uso regular de crack e/ ou similares (Bastos \& Bertoni, 2014) encontrou uma estimativa de usuários de drogas ilícitas de 2,28\% (com exceção da maconha), dos quais $0,81 \%$ eram usuários regulares de crack e/ou similares.
Embora percentualmente baixo quando comparado às drogas legalizadas, os usuários de crack representaram 35\% (370 mil pessoas) da amostra de usuários de drogas ilícitas.

O consumo de crack tem sido alvo de ações da saúde pública tanto pela prevalência de usuários, quanto por estar associado a graves problemas de ordem física, social e psiquiátrica (Santos, Neto, \& Sousa, 2012). Em comparação aos usuários de cocaína em pó, estudos mostram que usuários de crack 
apresentam maior envolvimento com atividades ilegais, troca de sexo por droga, efeitos adversos mais graves, maiores chances de morar ou ter morado na rua (Sanchez \& Nappo, 2007) e maiores prejuízos psiquiátricos e sociais (Duailibi, Ribeiro, \& Laranjeira, 2008; Humeniuk \& Poznyak, 2004).

Nos últimos anos, a divulgação realizada pela mídia dos problemas associados ao crack está voltada aos atos de violência e criminalidade praticados e sofridos pelos usuários (Romanini \& Roso, 2013). Estudos que focaram a relação da mídia com o crack mostraram que, em muitos casos, as reportagens são elaboradas de forma sensacionalista e embasadas no discurso antidrogas (Romanini \& Roso, 2013; Santos, Neto, \& Sousa, 2012). Por outro lado, no ambiente acadêmico há pesquisas que de fato correlacionam a violência e a criminalidade com o consumo de crack (Carvalho \& Seibel, 2009; Giannini, Miller, Loiselle, \& Turner, 1993; Kessler, Terra, Faller, Stolf, Peuker, \& Benzano, 2012; Vaughn, Fu, Perron, Bohnert, \& Howard, 2010). O levantamento epidemiológico nacional realizado por Bastos e Bertoni (2014) mostrou que quase metade dos usuários de crack/similares havia sido presa pelo menos uma vez na vida, principalmente devido ao uso ou posse de drogas (13,9\%), assalto/roubo (9,2\%), furto/fraude/invasão de domicílio $(8,5 \%)$ e tráfico ou produção de drogas (5,5\%). Carvalho e Seibel (2009) avaliaram as práticas de uso de crack e seu envolvimento com a violência em uma amostra de 350 usuários de drogas em tratamento no estado de São Paulo. Os resultados mostraram que a violência foi relatada por $97 \%$ dos participantes do estudo. Atos de violência, como agressões verbais e físicas, ameaças de morte, roubo e tráfico de drogas foram significativamente maiores entre usuários de crack. Um grande número de usuários de crack tinha histórico de prisão anterior, muitas delas ocasionadas por infrações relacionadas às drogas. Em um levantamento nacional americano sobre usuários de crack que não estavam em tratamento, Vaughn, Fu, Perron, Bohnert e Howard (2010) encontraram um maior envolvimento com criminalidade quando comparados aos de cocaína em pó. No entanto, os autores reforçam a necessidade da realização de mais estudos nessa área a fim de esclarecer essa relação.

Além da identificação da prevalência e histórico criminal, faz-se necessário compreender quais são os fatores que estão atual e historicamente associados à violência e criminalidade entre usuários de crack. Embora exista uma literatura crescente sobre os usuários de crack nos últimos anos, e esteja disponível alguma informação sobre seu envolvimento com a criminalidade, poucos estudos se propõem a estudar os fatores associados a esse fenômeno (Guimarães, Santos, Freitas, \& Araújo, 2008). Oser et al. (2011) investigaram usuários de estimulantes, entre eles o crack, em comunidades rurais americanas que não estavam em tratamento para determinar os fatores associados às prisões por crimes relacionados com a substância, crimes contra a propriedade e à detenção por outros crimes. Os resultados de três modelos de regressão logística indicaram que usuários de estimulantes mais jovens, aqueles com mais condenações e aqueles que usavam crack com frequência eram significativamente mais propensos a terem sido presos por crime relacionado com a substância, crime contra a propriedade, ou outro crime nos seis meses anteriores ao estudo. Há evidências na literatura de alguns fatores que aumentam a vulnerabilidade dos usuários de substâncias para consequências mais graves. Estudos mostram que a idade de início de uso de substâncias está associada a problemas de saúde pública (Ellickson, Tucker, \& Klein, 2003) dentre eles o encarceramento (Slade et al., 2008), que usuários de crack apresentam maiores problemas familiares quando comparados a outras drogas (Moura, Benzano, Pechansky, \& Kessler, 2014), que problemas psiquiátricos e renda menor (Vaughn et al., 2010) estão associados ao aumento da criminalidade entre usuários de crack quando comparados aos usuários de cocaína, e que usuários de crack em tratamento no Brasil apresentaram mais problemas familiares, de emprego e com a justiça criminal do que usuários de cocaína em pó (Kessler, Terra et al., 2012). No entanto, não foram encontrados estudos que explorassem a associação de todos esses fatores à criminalidade e violência entre dependentes de crack em tratamento.

A identificação do envolvimento com a criminalidade e dos fatores associados podem trazer importantes contribuições para ações de políticas públicas, incluindo aquelas voltadas para a prevenção e tratamento dos usuários de crack. A gravidade dos problemas associados ao crack tem fomentado a discussão sobre a melhor abordagem para o tratamento dos dependentes desta substância (Kessler \& Pechansky, 2008). Um levantamento realizado em 2007 mostrou que $67 \%$ das instituições para tratamento dos usuários de substâncias no Brasil são não governamentais, dentre elas as Comunidades Terapêuticas (CTs). As CTs são instituições privadas que têm obtido um destaque atual como importante aliadas da rede de atenção ao 
usuário de drogas do Sistema Único de Saúde (SUS) (Alves, 2009) e têm sido apontadas como uma possibilidade de tratamento para usuários dessa substância (Kessler \& Pechansky, 2008). Estudos têm indicado que grande parte dos pacientes que buscam tratamento atualmente o fazem devido ao uso de crack (Duailibi et al., 2008), incluindo nas CTs (Madalena, 2013). Porém, pouco se sabe a respeito da população atendida nessas instituições (Scaduto, Barbieri, \& Santos, 2014) e sobre o envolvimento dela com criminalidade e violência.

Dessa forma, o presente estudo teve como objetivo avaliar a criminalidade e violência entre dependentes de crack em tratamento em CTs, buscando especificamente: descrever o histórico com a justiça criminal e envolvimento recente com criminalidade e violência; e identificar os fatores associados à prisão após os 18 anos e a criminalidade e violência nos últimos seis meses.

\section{Método}

Este estudo é descritivo, de natureza quantitativa e delineamento transversal. Trata-se de um recorte de um estudo maior que avaliou usuários de crack em tratamento em Comunidades Terapêuticas.

\section{Participantes}

Foram incluídos no estudo 72 indivíduos que preenchiam aos critérios de inclusão: ter mais do que 18 anos, estar em tratamento há no máximo 30 dias em três CTs de uma cidade de porte médio de Minas Gerais e preencher critérios indicativos de dependência de crack de acordo com a pontuação obtida no Teste de Triagem do Envolvimento com Álcool, Cigarro e Outras Substâncias (ASSIST). Foram excluídos 11 indivíduos por não preencherem os critérios de inclusão, ou por não terem aceitado terminar a entrevista.

\section{Instrumentos}

ASI 6 (Addiction Severity Index versão 6) (Kessler, Cacciola et al., 2012) - Trata-se de uma entrevista semiestruturada composta por 242 questões agrupadas em sete domínios: problemas médicos, de emprego/suporte, com uso de álcool e outras drogas, legais, sociofamiliares (relacionamento com adultos, trauma, convívio com crianças) e psiquiátricos. Seus itens trazem informações sobre histórico de problemas e de tratamento em cada área, bem como problemas ocorridos nos últimos seis meses e 30 dias anteriores à entrevista. Por se tratar de um estudo sobre crack, questões sobre essa substância foram separadas da classe de cocaína na área sobre drogas. Foram utilizados os escores de gravidade de problemas que são definidos pela percepção do entrevistado quanto a sua necessidade de tratamento/aconselhamento para cada um dos domínios. As questões da área de problemas legais foram utilizadas para descrição do histórico e envolvimento com a criminalidade e violência.

ASSIST (Henrique, Micheli, Lacerda, Lacerda, \& Formigoni, 2004) - Questionário estruturado contendo oito questões sobre o padrão de consumo de nove classes de substâncias psicoativas (tabaco, álcool, maconha, cocaína, estimulantes, sedativos, inalantes, alucinógenos e opiáceos). As questões sobre crack também foram desvinculadas da classe de cocaína.

Questionário sociodemográfico - Questionário estruturado contendo a Escala Socioeconômica (ABIPEME), além de informações sobre idade, sexo, escolaridade, emprego e religião.

\section{Procedimentos}

A coleta de dados foi realizada entre agosto de 2012 a maio de 2013, totalizando nove meses, nas próprias CTs. Os indivíduos foram convidados a participar de uma entrevista com duração de aproximadamente 90 minutos, utilizando-se os instrumentos padronizados. Todos os participantes assinaram um Termo de Consentimento Livre e Esclarecido redigido de acordo com os critérios do Comitê de Ética em Pesquisa da Universidade Federal de Juiz de Fora, que aprovou este projeto ( $\left.{ }^{\circ} 131157\right)$. A aplicação dos instrumentos foi realizada em um local isolado, estando presentes somente o entrevistador e o entrevistado. Os entrevistadores foram devidamente treinados para aplicação dos instrumentos, especialmente o ASI 6.

\section{Local do Estudo}

A coleta de dados foi realizada em três CTs, duas masculinas e uma feminina, escolhidas por conveniência dentre as regularizadas do município pela Agência Nacional de Vigilância Sanitária (ANVISA) de acordo com a Resolução RDC no 29 (RDC n $\left.{ }^{\circ} 29,2011\right)$. Todas as instituições seguiam uma abordagem terapêutica mista baseada na modalidade Minessota (12 passos) e espiritual. Elas contavam com profissionais de saúde de nível superior e monitores. A CT feminina - única da região - tinha capacidade para acolher 12 mulheres, tempo máximo de tratamento de seis meses que podia ser financiado pelo SUS ou particular. Uma das CTs masculinas possuía as mesmas características da 
feminina, porém com capacidade para 25 homens. A outra CT masculina, tinha capacidade para 35 homens, com tempo de duração possível de nove meses de tratamento e financiamento exclusivamente particular.

\section{Análise Estatística}

Para a análise descritiva foram calculados a média \pm desvio-padrão e frequência e porcentagem. Visando identificar fatores associados ao histórico de prisão após os 18 anos e ao envolvimento recente com a criminalidade, foram conduzidas uma série de análises exploratórias utilizando a correlação de Spearman. As variáveis escolhidas para as análises exploratórias foram feitas com base na literatura que identifica fatores comuns ao uso de drogas e ao envolvimento com a criminalidade. Dentre elas pode-se destacar: variáveis sociodemográficas, histórico de prisão anterior, idade de início de uso de cada droga e nível de gravidade de problemas em cada área do ASI 6.

As variáveis que se correlacionaram significativamente foram posteriormente incluídas em análises de regressão linear de Poisson para o cálculo das razões de risco (RR) visando testar a contribuição de cada variável em dois modelos distintos. O primeiro modelo buscou identificar as variáveis associadas ao número de prisões após os 18 anos (variável dependente), enquanto o segundo avaliou as variáveis associadas ao envolvimento recente com a criminalidade (variável dependente). Para isso, foi necessário criar três novas variáveis que foram incluídas nas regressões: a primeira variável se refere à soma de crimes seguidos por prisões/detenções cometidos antes dos 18 anos; a segunda variável se refere à soma do número de vezes em que o indivíduo foi preso/detido após os 18 anos, e a terceira variável é referente à soma de dias de envolvimento com crimes nos seis meses anteriores à entrevista.

\section{Resultados}

A tabela 1 apresenta os dados sociodemográficos dos participantes, os níveis de gravidade de problemas em cada área e a idade de início de uso de cada droga. Dos 72 indivíduos entrevistados, apenas 25,0\% eram mulheres. A média de idade dos usuários de rrack foi de 30 anos $(D P= \pm 7,2)$. A amostra era composta de indivíduos de cor parda/mestiça (41,7\%), que nunca se casaram $(60,6 \%)$, de religião protestante $(61,7 \%)$, que estavam desempregados $(65 \%)$ e que pertenciam às classes $\mathrm{B}(30,4 \%)$ ou $\mathrm{C}(50 \%)$. Observa-se que a primeira droga a ser consumida foi o álcool (14,3 \pm
3 anos), seguido pela maconha, inalantes, cocaína e o crack $(21,6 \pm 7,9$ anos).

Para avaliação dos níveis de gravidade de problemas foram utilizados escores de gravidade subjetivos de cada área do ASI 6. A partir das informações da entrevista, os participantes utilizavam uma escala do tipo Likert de cinco pontos $(0-$ nada a 4 -extremamente) que determinava a percepção do indivíduo quanto à necessidade de tratamento/aconselhamento em cada área no último mês. As médias foram consideradas altas se estavam mais próximas de 4 e baixas se estavam próximas a 0 . Os participantes tiveram médias altas quanto a problemas com drogas $(3,9 \pm 0,6)$, de emprego $(3,0$ $\pm 1,4)$ e psiquiátricos $(3,1 \pm 1,5)$. Na área sociofamiliar, a média foi alta para problemas de relacionamento com adultos $(3,1 \pm 1,3)$ e moderadas para convívio com crianças $(2,0 \pm 1,8)$ e traumas $(2,4 \pm 1,7)$. Quando comparada aos demais domínios, a média obtida na área de problemas legais foi baixa $(0,5 \pm 1,1)$.

A tabela 2 mostra o histórico de prisões e detenções, bem como os motivos das prisões ocorridas antes e após os 18 anos e nos últimos seis meses. Além disso, apresenta a situação recente com a justiça criminal. Observou-se que a maioria dos usuários foi preso/ detido em algum período da vida ainda que por poucas horas $(76,4 \%)$, sendo que $35,0 \%$ deles ficaram presos por mais tempo. Somente $9,7 \%$ dos indivíduos foram condenados e presos antes dos 18 anos, dentre eles, 9,1\% foram presos por ato ilícito ligado a drogas e 7,5\% por crimes visando lucro ou contra propriedade. Pode-se observar também que a média de idade da primeira prisão foi baixa (22,3 anos).

Quanto às prisões e detenções ocorridas após os 18 anos, ressalta-se que 29,2\% dos entrevistados informaram terem sido presos ao menos uma vez neste período. Quase $50 \%$ deles foram presos ou detidos por motivos relacionados ao porte, venda ou produção de drogas. Nos últimos seis meses, também houve relatos de prisões, causadas principalmente pela venda ou produção de drogas $(6,2 \%)$ e roubo $(6,2 \%)$. Já com relação ao envolvimento com a justiça criminal, $8,3 \%$ dos participantes estavam sendo investigados em inquérito policial, $11,1 \%$ estavam aguardando julgamento ou sentença e $11,1 \%$ estavam em liberdade condicional no período da entrevista.

A tabela 3 apresenta situações de criminalidade e violência recentes, ocorridas nos últimos seis meses e nos últimos 30 dias anteriores à entrevista. Dentre os 72 participantes, $57(79,2 \%)$ informaram terem se envolvido ao menos um dia com qualquer atividade 
Tabela 1

Dados Sociodemográficos dos Usuários de Crack em Tratamento em Comunidades Terapêuticas $(N=72)$

\begin{tabular}{|c|c|}
\hline Perfil Sociodemográfico & Usuários de Crack $(N=72)$ \\
\hline \multicolumn{2}{|l|}{ Gênero } \\
\hline masculino & $54(75,0)$ \\
\hline feminino & $18(25,0)$ \\
\hline \multirow{2}{*}{ Idade } & $30,0 \pm 7,2$ \\
\hline & (20 - 50 anos) \\
\hline \multicolumn{2}{|l|}{ Raça } \\
\hline negra/preta & $17(23,6)$ \\
\hline branca & $16(22,2)$ \\
\hline parda/mestiça & $30(41,7)$ \\
\hline \multicolumn{2}{|l|}{ Estado Civil } \\
\hline vivendo como/casado & $16(822,6)$ \\
\hline nunca casou & $43(60,6)$ \\
\hline \multicolumn{2}{|l|}{ Religião } \\
\hline protestante & $29(61,7)$ \\
\hline católico & $12(25,5)$ \\
\hline nenhuma & $3(6,4)$ \\
\hline \multicolumn{2}{|l|}{ Nível de escolaridade } \\
\hline nenhum & $15(20,8)$ \\
\hline ensino fundamental & $33(45,8)$ \\
\hline ensino médio & $21(29,2)$ \\
\hline ensino superior & $2(2,28)$ \\
\hline Trabalha & $25(34,7)$ \\
\hline \multicolumn{2}{|l|}{ Escala Social } \\
\hline A1/A2 & $4(5,5)$ \\
\hline B1/B2 & $22(30,4)$ \\
\hline $\mathrm{C} 1 / \mathrm{C} 2$ & $36(50)$ \\
\hline $\mathrm{D} / \mathrm{E}$ & $10(13,9)$ \\
\hline
\end{tabular}

Idade de início de uso

álcool

$14,3 \pm 3$

\begin{tabular}{l} 
álcool \\
\hline maconha \\
\hline inalantes \\
\hline cocaína \\
\hline crack \\
\hline Nível de gravidade de problemas no último mês \\
álcool \\
drogas \\
saúde \\
emprego \\
Problemas de relacionamento com adultos \\
Traumas \\
Convívio com crianças \\
Psiquiátrica
\end{tabular}

$18,5 \pm 7$ (12 - 46 anos) $21,6 \pm 7,9$ (13 - 46 anos)

Nota. $* 0$ - nenhum; 1 - leve; 2 - moderado; 3 - considerável; 4 - extremo

$2,2 \pm 1,8$

$3,9 \pm 0,6$

$2,4 \pm 1,7$

$3,0 \pm 1,4$

$3,1 \pm 1,3$

$2,4 \pm 1,7$

$2,0 \pm 1,8$

$3,1 \pm 1,5$ 
Tabela 2

Prisões e Envolvimento dos Usuários de Crack $(N=72)$ com a Justiça Criminal

\begin{tabular}{|c|c|c|c|}
\hline Ações de crime ou violência & $\mathrm{Na}$ vida & & \\
\hline Detido ou preso por poucas horas - $\mathrm{n}^{\circ}(\%)$ & $55(76,4)$ & & \\
\hline Preso $-\mathrm{n}^{\circ}(\%)$ & $28(35,0)$ & & \\
\hline \multirow[t]{2}{*}{ Idade da primeira prisão } & $22,3 \pm 6,6$ & & \\
\hline & $\begin{array}{c}\text { Antes dos } 18 \\
\text { anos }\end{array}$ & $\begin{array}{c}\text { Após os } 18 \\
\text { anos }\end{array}$ & $\begin{array}{c}\text { Últimos } 6 \\
\text { meses }\end{array}$ \\
\hline $\mathrm{N}^{\mathrm{o}}$ pessoas presas $-\mathrm{n}^{\mathrm{o}}(\%)$ & $7(9,7)$ & $21(29,2)$ & - \\
\hline $\mathrm{N}^{o}$ vezes condenado & $0,2 \pm 1$ & $0,3 \pm 0,6$ & - \\
\hline \multicolumn{4}{|l|}{ Motivos prisão } \\
\hline Crimes violentos ou contra pessoas - $\mathrm{n}^{\mathrm{o}}(\%)$ & $2(3,8)$ & - & - \\
\hline Ato ilícito ligado a drogas - $\mathrm{n}^{\circ}(\%)$ & $5(9,1)$ & - & - \\
\hline Crimes visando lucro ou contra propriedade - $\mathrm{n}^{\circ}(\%)$ & $4(7,5)$ & - & - \\
\hline Infrações praticadas somente por jovens - $\mathrm{n}^{\circ}(\%)$ & $1(1,9)$ & - & - \\
\hline Porte de drogas - $\mathrm{n}^{\circ}(\%)$ & - & $23(35,4)$ & $2(3,1)$ \\
\hline Venda ou produção de drogas - $\mathrm{n}^{\circ}(\%)$ & - & $9(14,1)$ & $4(6,2)$ \\
\hline Roubo - $\mathrm{n}^{\circ}(\%)$ & - & $10(14,9)$ & $4(6,2)$ \\
\hline Outros crimes visando lucro - $\mathrm{n}^{\circ}(\%)$ & - & $8(12,3)$ & $2(3,1)$ \\
\hline Crime violento - $\mathrm{n}^{\mathrm{o}}(\%)$ & - & $13(19,7)$ & $1(1,5)$ \\
\hline Armas, prostituição ou jogo - no (\%) & - & $3(4,6)$ & 0 \\
\hline Dirigir alcoolizado - $\mathrm{n}^{\circ}(\%)$ & - & $2(3,1)$ & 0 \\
\hline Admissão para o tratamento ordenada pela justiça - $\mathrm{n}^{\circ}(\%)$ & - & - & $1(1,4)$ \\
\hline Investigado em inquérito policial - $\mathrm{n}^{\mathrm{o}}(\%)$ & - & - & $6(8,3)$ \\
\hline Suspensão condicional do processo - $\mathrm{n}^{\mathrm{o}}(\%)$ & - & - & 0 \\
\hline Aguardando julgamento ou sentença - $\mathrm{n}^{\mathrm{o}}(\%)$ & - & - & $8(11,1)$ \\
\hline Sursis ou em Liberdade condicional - $\mathrm{n}^{\mathrm{o}}(\%)$ & - & - & $8(11,1)$ \\
\hline Participando de um programa de justiça terapêutica - $n^{\circ}(\%)$ & - & - & $2(2,8)$ \\
\hline
\end{tabular}

criminal ou violência. Observa-se que, nos últimos seis meses, 33,8\% dos usuários de crack venderam ou fabricaram drogas, 30\% furtaram alguma coisa, venderam objetos roubados, falsificaram prescrições ou cheques, destruíram propriedade ou incendiaram algo, e $28,6 \%$ roubaram alguém. Além disso, 25\% carregaram arma sem licença e $27,1 \%$ dirigiram sob efeito de drogas ou álcool. A média de dias em que cometeram atos ilícitos nos últimos seis meses foi moderada nos crimes relacionados a vender ou fabricar drogas (24,1 dias), carregar armas sem licença (14,2 dias) e dirigir sob efeito de drogas ou álcool (13,3 dias). Com relação aos últimos 30 dias, as porcentagens de usuários envolvidos em situações de criminalidade foram moderadas, destacando furtar alguma coisa, vender objetos roubados, falsificar prescrições ou cheques, destruir propriedade ou incendiar algo $(23,5 \%)$ e roubar $(20,9 \%)$.

Os dados referentes à prática de violência mostraram que $41,7 \%$ dos usuários de crack ameaçaram ou agrediram alguém nos últimos seis meses, e $22,2 \%$ o fizeram nos últimos 30 dias. Nos últimos seis meses, $29,6 \%$ dos usuários ameaçaram sem agressão física e $28,2 \%$ agrediram fisicamente sem uma arma. A média de dias em que ocorreu violência nos últimos seis meses foi baixa, em torno de 3,6 dias.

Para testar os fatores associados às prisões ocorridas após os 18 anos e aqueles associados ao envolvimento com a criminalidade nos últimos seis meses, foram realizadas duas regressões de Poisson (tabela 4). No primeiro modelo, nossas hipóteses eram 
Tabela 3

Envolvimento Recente dos Usuários de Crack $(N=57)$ com Situações de Criminalidade ou Violência

\begin{tabular}{|c|c|c|c|c|}
\hline Ações de crime ou violência & $\begin{array}{l}\text { Últimos } 6 \\
\text { meses } \\
\mathrm{n}^{\mathrm{o}}(\%)\end{array}$ & $\mathrm{N}^{\circ}$ dias & $\begin{array}{l}\text { Últimos } 30 \\
\quad \text { dias } \\
\mathrm{n}^{\circ}(\%) \\
\end{array}$ & $\mathrm{N}^{\circ}$ dias \\
\hline Vendeu ou fabricou drogas & $24(33,8)$ & $24,1 \pm 47,4$ & $12(17,6)$ & $3,0 \pm 7,8$ \\
\hline Roubou alguém & $20(28,6)$ & $7,6 \pm 29,3$ & $14(20,9)$ & $0,6 \pm 1,8$ \\
\hline $\begin{array}{l}\text { Furtou alguma coisa, vendeu objetos roubados, } \\
\text { falsificou prescrições ou cheques, destruiu } \\
\text { propriedade ou incendiou algo }\end{array}$ & $28(30,0)$ & $6,8 \pm 25,4$ & $16(23,5)$ & $0,9 \pm 2,3$ \\
\hline Roubou em loja & $7(9,9)$ & $2 \pm 14,3$ & & \\
\hline Praticou arrombamento & $5(7,0)$ & $0,2 \pm 0,9$ & & \\
\hline Roubou veículo a motor & $1(1,4)$ & $0,0 \pm 0,1$ & & \\
\hline Falsificou & $2(2,8)$ & $0,1 \pm 0,6$ & & \\
\hline Fraudou & $0(0)$ & $0,0 \pm 0,0$ & & \\
\hline Cometeu vandalismo & $5(7,0)$ & $0,2 \pm 1$ & & \\
\hline Cometeu incêndio (premeditado) & $4(5,6)$ & $0,1 \pm 0,4$ & & \\
\hline Roubou/danificou propriedade & $9(12,7)$ & $2,7 \pm 17,9$ & & \\
\hline Ameaçou ou agrediu alguém & $30(41,7)$ & $3,6 \pm 10,6$ & $16(22,2)$ & $0,4 \pm 0,8$ \\
\hline Ameaçou sem agressão física & $21(29,6)$ & $1,5 \pm 4,1$ & & \\
\hline Agrediu fisicamente com uma arma & $7(10,0)$ & $0,4 \pm 1,9$ & & \\
\hline Agrediu fisicamente sem uma arma & $20(28,2)$ & $1,9 \pm 7$ & & \\
\hline Agrediu sexualmente & $1(1,4)$ & $0,0 \pm 0,1$ & & \\
\hline Assassinou alguém & $2(2,8)$ & $0,1 \pm 0,4$ & & \\
\hline Carregou uma arma sem licença & $16(25,0)$ & $14,2 \pm 42,1$ & & \\
\hline Envolveu-se com prostituição/cafetinagem & $8(12,7)$ & $4,2 \pm 23,2$ & & \\
\hline Jogo ilegal & $4(6,3)$ & $3,3 \pm 22,8$ & & \\
\hline Dirigiu sob efeito de drogas ou álcool & $19(27,1)$ & $13,3 \pm 36,9$ & $13(21,7)$ & $2,6 \pm 7,4$ \\
\hline
\end{tabular}

que um menor nível educacional, ser homem, ter sido preso antes dos 18 anos e a idade de início de uso de qualquer droga precocemente iriam aumentar as chances de prisão após os 18 anos. Todas essas variáveis foram testadas visando encontrar o melhor modelo de associação. O melhor modelo encontrado mostrou que ser homem aumenta em 4,19 vezes a chance de ter sido preso após os 18 anos; ter sido preso antes dos 18 anos aumenta em $15 \%$ a chance de ter sido preso novamente após os 18; cada nível de instrução estava associado à redução de 55\% na chance de prisão após os 18 anos; e cada ano a menos de início de uso de álcool na adolescência aumentou em 14\% a chance de ser preso após os 18 anos. Vale destacar que análises anteriores testando a idade de início de uso de crack $(\mathrm{RR}=0,978-$ IC 95\%
$0,913-1,05, p=0,530)$, de maconha $(\mathrm{RR}=1,0-\mathrm{IC} 95 \%$ $0,951-1,06, p=0,877)$ e de qualquer droga $(\mathrm{RR}=0,978$ - IC 95\% 0,913-1,05, $p=0,530$ ) em substituição à idade de início do uso de álcool não obtiveram efeitos significativos no modelo.

Para o segundo modelo, as hipóteses eram que um menor nível educacional, ser homem, apresentar problemas psiquiátricos, problemas sociofamiliares e de emprego, ter sido preso antes e após 18 anos e a idade de início do uso de qualquer droga estaria associado ao envolvimento recente com a criminalidade. Após serem testados modelos incluindo essas variáveis, o melhor modelo mostrou que ser homem reduziu em $12 \%$ a chance de envolvimento recente com crime; cada nível de instrução a mais reduziu em 33\% esta chance. 
Tabela 4

Relação entre Fatores Sociodemográficos, Histórico de Prisão, Idade de Início de Uso de Drogas e Perfil de Gravidade com Prisão após os 18 Anos e Criminalidade/Violência nos Últimos 6 Meses

\begin{tabular}{|c|c|c|}
\hline Fatores & $\mathrm{RR}(\mathrm{IC} \%)$ & $p$ \\
\hline \multicolumn{3}{|c|}{ Modelo 1 Fatores associados à prisão após os 18 anos } \\
\hline Gênero (se homem) & $4,19(1,93-9,01)$ & 0,001 \\
\hline Nível de instrução (se maior) & $0,45(0,35-0,57)$ & 0,001 \\
\hline $\mathrm{N}^{\circ}$ prisões antes dos 18 anos (se maior) & $1,15(1,03-1,30)$ & 0,012 \\
\hline Idade de início de uso de álcool (se mais velho) & $0,86(0,81-0,92)$ & 0,001 \\
\hline \multicolumn{3}{|c|}{ Modelo 2 Fatores associados à criminalidade/ violência nos últimos 6 meses } \\
\hline Gênero (se homem) & $0,88(0,81-0,95)$ & 0,001 \\
\hline Nível de instrução (se maior) & $0,77(0,76-0,80)$ & 0,001 \\
\hline Problemas com emprego (se maior) & $1,62(1,56-1,68)$ & 0,001 \\
\hline Problemas psiquiátricos (se maior) & $1,06(1,03-1,08)$ & 0,001 \\
\hline $\mathrm{N}^{\circ}$ prisões antes dos 18 anos (se maior) & $1,09(1,06-1,14)$ & 0,001 \\
\hline
\end{tabular}

Por outro lado, a percepção de problemas atuais com emprego aumentou em $62 \%$ a chance de envolvimento recente com crime, e a percepção maior de ter problemas psiquiátricos aumentou em $6 \%$. O número de prisões antes dos 18 anos aumentou em $9 \%$ a chance de envolvimento recente com o crime.

\section{Discussão}

Diante da preocupação de profissionais de saúde e da comunidade acadêmica e sociedade em torno do uso de crack no Brasil, dos problemas psicossociais associados ao crack e do papel que as CTs têm ocupado nas políticas públicas no país, este estudo teve como objetivos descrever o histórico e o envolvimento recente dos usuários de crack em tratamento em CTs com a violência e a criminalidade, bem como identificar fatores associados ao histórico de prisão e envolvimento recente com a criminalidade/violência. Quanto ao histórico de problemas com a justiça criminal, observou-se que a maioria dos usuários de crack esteve envolvida em algum momento na vida com situações de criminalidade, que geraram como consequência a prisão ou detenção, ainda que por poucas horas. Esse dado corrobora resultados de outros estudos que investigaram o histórico de prisões ou detenções em usuários de crack (Bastos \& Bertoni, 2014; Dudish \& Hatsukami, 1996; Guimarães et al., 2008). A prevalência de prisões propriamente ditas (35\%) foi semelhante à de 33,3\% encontrada por Guimarães, Santos, Freitas e Araújo,
(2008) em uma unidade de internação para desintoxicação no Rio Grande do Sul. Dentre os presos no estudo, uma parcela menor relatou ter sido presa antes dos 18 anos por motivos diversos ligados à crimes violentos, problemas com drogas e visando lucro. Porém, cerca de um terço da amostra foi presa após os 18 anos por motivos associados especialmente a atividades ilegais relacionadas a drogas, roubo e crimes violentos. Esses resultados são consistentes com os encontrados por Oser et al. (2011).

O primeiro modelo de análise de regressão logística buscou identificar os fatores associados ao número de prisões ocorridas após os 18 anos. A hipótese quanto ao gênero foi corroborada indicando que ser homem estava associado a um maior número de prisões. Esse dado também tem sido observado em outros estudos (Carvalho \& Seibel, 2009; Vaughn et al., 2010). A prisão ocorrida antes dos 18 anos também aumentou a chance de ocorrência de prisão após os 18 anos. Embora não seja possível estabelecer uma relação de causalidade neste estudo, esses dados indicam que a prisão na adolescência não agiu como um fator de proteção ao envolvimento futuro com a criminalidade associada a novas prisões. Esse aspecto não deve ser negligenciado, visto que a discussão a respeito da redução da maioridade penal como uma proposta de política pública para redução da violência vem surgindo em função da preocupação da sociedade com a violência e da responsabilização de jovens (Almeida \& Sousa, 2010). Por outro lado, nossas hipóteses de que 
as prisões após os 18 anos estariam associadas à idade de início de uso de crack ou de qualquer droga ilícita não foram corroboradas. Da mesma forma, não foi encontrada associação com relação à idade de início de uso de qualquer droga ilícita, mas sim com o uso de álcool. É provável que essa associação seja decorrência de a idade de início de uso de álcool ser a menor na amostra estudada, indicando ser a primeira droga consumida. Esse resultado corrobora estudos longitudinais que associam a idade de início de uso de álcool a problemas de saúde pública (Ellickson et al., 2003) dentre eles o encarceramento Slayer (Slade et al., 2008). Esses dados enfatizam a necessidade de se evitar o início precoce do consumo de qualquer substância, inclusive as lícitas.

Como hipotetizado, o maior nível de instrução foi um importante protetor, reduzindo a chance da ocorrência de prisões após os 18 anos, o que corrobora o encontrado em outros estudos (Vaughn et al., 2010). Vale destacar que a amostra estudada das CTs possuía um maior nível socioeconômico, sendo que mais de $70 \%$ pertencia à classe média, o que pode explicar o maior nível educacional. Esse dado difere do que é encontrado em amostras de levantamentos epidemiológicos ou em amostras de serviços exclusivamente públicos (Bastos \& Bertoni, 2014; Garcia, Zacharias, Winter, \& Sontag, 2012). Por um lado, esse resultado mostra que a criminalidade entre usuários de crack não está associada somente a pessoas de baixa renda. Por outro, salienta que o investimento na educação dos indivíduos como fator de prevenção é importante e que, para aqueles que estão em tratamento, vale a pena investir na reinserção educacional, especialmente dos mais jovens.

Em relação ao envolvimento recente dos usuários com a criminalidade e violência, a grande maioria da amostra relatou o envolvimento com crimes nos últimos seis meses. Os usuários entrevistados relataram terem participado principalmente de roubos, furtos e crimes relacionados ao tráfico de drogas (venda ou produção de drogas), tanto após os 18 anos, quanto nos últimos seis meses. Beato Filho et al. (2001) descreveram em um estudo sobre o número de homicídios em Belo Horizonte (MG) que a violência estava associada a um perfil econômico e social baixo, bem como ao tráfico de drogas. Werb, Kerr, Li, Montaner e Wood (2008) investigaram os fatores associados ao tráfico de drogas entre jovens de rua. Eles descreveram que 58\% da amostra relatou ter se envolvido com tráfico de drogas nos últimos seis meses, sendo que $85,6 \%$ disseram que a principal razão de vender drogas era para pagar o seu uso pessoal. Como no presente estudo grande parcela da amostra era composta por pessoas de classe média, é possível que o envolvimento com o tráfico não estivesse diretamente relacionado ao baixo perfil econômico, mas à manutenção do consumo. Além disso, uma parcela importante da amostra de dependentes de crack estudada relatou envolvimento com atividades violentas, como ter ameaçado ou agredido alguém nos últimos seis meses. Outros estudos têm relatado a associação entre violência e uso de crack (Giannini, et al., 1993; Miller, Gold, \& Mahler, 1990; Vaughn et al., 2010). No Brasil, Kessler, Terra et al. (2012) também encontraram entre usuários de crack em tratamento em cinco estados altas taxas de envolvimento com roubo, furto, ameaças e agressões. Goldstein (1985) propôs que drogas e violência estão relacionadas de três formas possíveis: (1) psicofarmacologicamente, na qual as pessoas podem se envolver em atos irracionais ou violentos, como resultado dos efeitos de curto ou longo prazo do uso de drogas; (2) econômico-compulsivo, em que alguns usuários de drogas cometem crimes violentos orientados economicamente, a fim de sustentar seu uso de drogas e (3) sistêmica, referindo-se aos padrões tradicionalmente violentos de interação envolvidos no sistema de distribuição de drogas. No presente estudo, não foi possível identificar se os atos de violência citados pelos participantes foram praticados durante o efeito do consumo de crack. Porém, devido à quase metade dos usuários ter se envolvido com situações de criminalidade ligadas ao porte, venda ou produção de drogas, pode-se identificar que a perspectiva sistêmica citada por Goldstein (1985) estava presente.

No segundo modelo avaliado neste estudo, tinha-se como hipóteses que fatores sociodemográficos, como ser homem e ter maior nível de instrução, o histórico de prisões antes e após os 18 anos, a idade de início de uso de drogas e problemas recentes de emprego, psiquiátricos e de relacionamento familiar estariam associados ao envolvimento recente com a criminalidade e violência. Nesse caso, a hipótese quanto ao sexo não foi corroborada, pois ser mulher estava associado ao aumento de chance de envolvimento recente com crime e violência. Estudos mostram que o consumo de crack entre mulheres tem aumentado. Um estudo longitudinal realizado em serviços públicos brasileiros mostrou que, dentre as 761 mulheres avaliadas, a taxa de hospitalizações relacionadas ao uso de crack aumentou de 2,8\% em 1997 para 67,8\% em 2010 (Moreira, Barbosa, Laranjeira, \& Mitsuhiro, 2014). Carvalho e Seibel (2009) relatam um maior envolvimento da mulher usuária de crack com situações de prostituição 
e troca de sexo por droga, porém no presente estudo a maior parte da amostra estava envolvida em roubo, furto e tráfico de drogas. Para Barcinski (2009), o conhecimento sobre o envolvimento de mulheres com a criminalidade é negligenciado, em geral, associado à criminalidade masculina. Em um estudo qualitativo, a autora entrevistou oito mulheres com histórico de tráfico de drogas no Rio de Janeiro. Os resultados sugerem que questões relacionadas à gênero, cor e classe permeiam a entrada, permanência e saída das mulheres no tráfico. Este estudo não teve como propósito principal estudar as diferenças de gênero ou sexo, porém vale considerar a importância de pensar em ações preventivas e de tratamento específicas para mulheres. Embora em levantamentos epidemiológicos a prevalência de mulheres usuárias de crack e outras drogas seja alta, são raros os tratamentos específicos. Cabe ressaltar que a CT estudada é a única da região específica para essa população que apresenta características singulares.

Como no modelo anterior, o nível de instrução agiu como um fator de proteção ao envolvimento recente com a criminalidade. O histórico de prisões anteriores aos 18 anos também se repetiu como risco, ainda que com um efeito menor. Nesse caso, além de a prisão na adolescência não surgir como um fator de proteção à manutenção de atividades ilegais, ainda, a prisão ocorrida após os 18 anos não esteve relacionada nem como proteção nem como risco.

Quanto à gravidade de problemas recentes, destacou-se nesse modelo que os problemas atuais com o emprego foi um importante fator associado à criminalidade. Mais de $65 \%$ da amostra estudada encontrava-se desempregada no momento da entrevista, correspondente ao encontrado na literatura (Carvalho \& Seibel, 2009). No estudo de Vaughn et al. (2010), uma renda menor estava associada a um aumento de 3,67 vezes a chance de ser usuário de crack ao invés de cocaína. Esse dado informa que o investimento na reinserção ocupacional dos usuários de crack em tratamento em CTs pode ser um fator de evitação de futuras atividades ilegais. Ele informa ainda que o envolvimento com atividades ilegais, principalmente tráfico e roubo, podem estar associado à manutenção do consumo. Por outro lado, os problemas psiquiátricos tiveram pouco efeito no crime recente, e os problemas de relacionamento familiar não interferiram no modelo.

Embora os problemas recentes relacionados a problemas psiquiátricos e sociofamiliares tenham tido menor ou nenhum efeito na criminalidade e violência recentes, observou-se de um modo geral que os participantes relataram que consideravam seus problemas de graves a moderados em todas as áreas do ASI 6, com exceção da área legal. Esses resultados sugerem que o tratamento dispensado aos pacientes deve ser proposto de forma abrangente, por equipe multiprofissional. No que tange à média baixa de problemas legais, pode-se ressaltar que, conforme observado nos resultados deste estudo, os usuários tiveram envolvidos com atividades ilegais recentemente, no entanto, não foram flagrados ou não estavam envolvidos com a justiça criminal. Nesse sentido, a percepção de gravidade quanto a problemas nessa área foi baixa. Kessler, Terra et al. (2012) avaliaram 738 usuários de drogas a fim de comparar usuários de cocaína em pó, cocaína em forma de crack e usuários de outras drogas em termos de comorbidades psiquiátricas e severidade de problemas psicossociais, dentre os resultados, observou-se que os usuários de crack apresentaram problemas significativamente maiores nas áreas legal, psiquiátrica e familiar quando comparados aos outros dois grupos avaliados.

\section{Considerações Finais}

Os resultados do presente estudo confirmam a literatura vigente de outras populações de usuários de crack, informando que os usuários em tratamento em CTs apresentam histórico de prisões e envolvimento recente com a criminalidade, principalmente roubo, furto e venda ou produção de drogas. Homens, pessoas com menor nível de instrução, ter iniciado o consumo de álcool precocemente e ter histórico de prisão na adolescência aumentam a chance de ter sido preso após os 18 anos. Ao passo que mulheres, sujeitos com menor nível de instrução, ter sido preso antes dos 18 anos e aqueles que apresentam maior gravidade de problemas recentes de emprego e psiquiátrico têm maior chance de envolvimento recente com a criminalidade e violência. Esse resultado aponta para a necessidade de se investir em políticas públicas que abordem a prevenção da criminalidade e da violência nessa população. Além disso, pode elucidar a realidade dos pacientes em tratamento em CTs, auxiliando no planejamento de ações de saúde pública direcionadas a essa forma de tratamento, dentre elas a reinserção ocupacional e educacional quando os indivíduos recebem alta das CTs.

A generalização dos resultados é restrita devido ao uso de amostra não probabilística. Outra limitação do estudo está relacionada ao tipo de coleta de dados. A entrevista face a face sobre experiências de violência pode ter o potencial viés de resposta de desejabilidade social. 
Estudos futuros podem incluir amostras probabilísticas de usuários de crack em tratamento em CTs, permitindo uma melhor exploração dos fatores associados ao envolvimento com o crime; devem investigar mais profundamente a relação entre criminalidade e uso de crack, tentando apontar por que os crimes foram cometidos, onde eles ocorreram e o perfil das vítimas. E, por fim, investigar as diferenças quanto ao gênero.

\section{Referências}

Almeida, R. O., \& Sousa, L. E. E. M. (2010). Liberdade assistida: Socioeducação e punição em conflito. O público e o privado, 15, 189-210. Recuperado de http:/ / seer.uece.br/?journal=opublicoeoprivado \&page $=$ article\&op $=$ view\&path $\% 5 B \% 5 \mathrm{D}=79$

Alves, V. S. (2009). Modelos de atenção à saúde de usuários de álcool e outras drogas: Discursos políticos, saberes e práticas. Cadernos de Saúde Pública, 25(11), 2309-2319. Recuperado de http://www.scielosp. $\mathrm{org} / \mathrm{pdf} / \mathrm{csp} / \mathrm{v} 25 \mathrm{n} 11 / 02 . \mathrm{pdf}$

Barcinski, M. (2009). Centralidade de gênero no processo de construção da identidade de mulheres envolvidas na rede do tráfico de drogas. Ciência \& Saúde Coletiva, 14(5), 1843-1853. doi: 10.1590/ S1413-81232009000500026

Bastos, F. I, \& Bertoni, N. [Org.] (2014). Pesquisa Nacional sobre o uso de crack. Recuperado de https:// www.icict.fiocruz.br/sites/www.icict.fiocruz.br/ files / Pesquisa $\% 20$ Nacional $\% 20$ sobre $\% 20$ o $\% 20$ Uso $\% 20$ de $\% 20$ Crack.pdf

Beato Filho, C. C., Assunção, R. M., Silva, B. F. A., Marinho, F. C. Reis, I. A., \& Almeida, M. C. M. (2001). Conglomerados de homicídios e o tráfico de drogas em Belo Horizonte, Minas Gerais, Brasil, de 1995 a 1999. Cadernos de Saúde Pública, 17(5), 11631171. doi: 10.1590/S0102-311X2001000500017

Carvalho, H. B., \& Seibel S. D. (2009). Crack cocaine use and its relationship with violence and HIV. Clinics, 64(9), 857-66. doi: 10.1590/ S1807-59322009000900006.

Cunha, P. J., Nicastri, S., Gomes, L. P., Moino, R. M, \& Peluso, M. A. (2004). Alterações neuropsicológicas em dependentes de cocaína/crack internados: Dados preliminares. Revista Brasileira de Psiquiatria, 26(1), 103-106. doi: 10.1590/ S1516-44462004000200007
Duailibi, L. B., Ribeiro, M., \& Laranjeira, R. (2008). Profile of cocaine and crack users in Brazil. Cadernos de Saúde Pública, 24(4), 545-557. doi: 10.1590/ S0102-311X2008001600007

Dudish, S. A., \& Hatsukami, D. K. (1996). Gender differences in crack users who are research volunteers. Drug and Alcobol Dependence, 42, 55-63. doi: 10.1016/0376-8716(96)01266-5

Ellickson, P.L., Tucker, J.S., Klein, D.J. (2003). Ten-year prospective study of public health problems associated with early drinking. Pediatrics, 111, 949-955. Recuperado de http://www.ncbi.nlm.nih.gov/ pubmed/12728070

Garcia, E. L., Zacharias, D. G, Winter, G., \& Sontag, J. (2012). (Re)Conhecendo o perfil do usuário de crack de Santa Cruz do Sul. Barbarói, 36, 83-95. Recuperado de http://online.unisc.br/seer/index. $\mathrm{php} /$ barbaroi/article/view/2922/2106\&ei=VzJf UPveLYbe9AS16oCAAg\&usg=AFQjCNEfbWt AcTrtI-8we79e7hjPKNhBxw

Giannini, A. J., Miller, N. S., Loiselle, R. H., \& Turner, C. E. (1993). Cocaine-associated violence and relationship to route of administration. Journal of Substance Abuse Treatment, 10, 61-69. doi: 10.1016/0740-5472(93)90101-7

Goldstein, P. J. (1985). The drugs/violence nexus: A tripartite conceptual framework. Journal of Drug Issues, 15, 493-506. Recuperado de http://www. drugpolicy.org/docUploads/nexus.pdf

Guimarães, C. F., Santos, D. V. V., Freitas, R. C., \& Araujo, R. B. (2008). Perfil do usuário de crack e fatores relacionados à criminalidade em unidade de internação para desintoxicação no Hospital Psiquiátrico São Pedro de Porto Alegre (RS). Revista de psiquiatria do Rio Grande do Sul, 30(2), 101-108. Recuperado de http://www.scielo.br/pdf/rprs/ v30n2/v30n2a05

Henrique, I. F. S., Micheli, D., Lacerda, R. B., Lacerda, L. A., \& Formigoni, M. L. O. S. (2004). Validação da versão brasileira do teste de triagem do envolvimento com álcool, cigarro e outras substâncias (ASSIST). Revista da Associação Médica Brasileira, 50(2), 199-206. Recuperado de http://www.scielo. $\mathrm{br} / \mathrm{pdf} / \mathrm{ramb} / \mathrm{v} 50 \mathrm{n} 2 / 20784 . p d f$

Humeniuk R, Poznyak V. (2004). Intervenção breve para o abuso de substâncias: Guia para uso na atenção primária à saúde. Genebra: OMS. 
Kessler, F. H. P., Cacciola, J., Alterman, A., Faller, S., Souza-Formigoni, M. L., Cruz, M. S., ... Pechansky, F. (2012). Psychometric properties of the sixth version of the Addiction Severity Index (ASI-6) in Brazil. Revista Brasileira de Psiquiatria, 34(1), 2433. Recuperado de http://www.ncbi.nlm.nih.gov/ pubmed/22392385

Kessler, F. H. P., \& Pechansky, F. (2008). Uma visão psiquiátrica sobre o fenômeno do crack na atualidade. Revista de psiquiatria do Rio Grande do Sul, 30(2), 96-98. Recuperado de http://www.scielo.br/pdf/ rprs/v30n2/v30n2a03.pdf

Kessler, F. H. P., Terra, M. B., Faller, S., Stolf, A. R., Peuker, A. C., \& Benzano, D. (2012). Crack Users Show High Rates of Antisocial Personality Disorder, Engagement in Illegal Activities and Other Psychosocial Problems. The American Journal on Addictions, 21, 370-380. doi: 10.1111/j.1521-0391.2012.00245.x

Madalena, T.S. (2013). Usuários de crack: Prevalência e perfil de pacientes em tratamento em comunidades terapêuticas (CTS) na cidade de Juiz de Fora, Minas Gerais (Dissertação de mestrado não publicada). Universidade Federal de Juiz de For a, Juiz de For a, Minas Gerais.

Miller, N. S., Gold, M. S., \& Mahler, J. C. (1990). A study of violent behaviors associated with cocaine use: Theoretical and pharmacological implications. Annals of Clinical Psychiatry, 2, 67-71. doi: 10.3109/10401239009150009

Moreira, M. M., Barbosa, G. L., Laranjeira, R., \& Mitsuhiro, S. S. (2014). Alcohol and crack cocaine use in women: A 14-year cross-sectional study. Journal of Addictive Diseases, 33(1), 9-14. doi: 10.1080/10550887.2014.882726

Moura, H. F, Benzano, D., Pechansky, F., \& Kessler, F. H. P. (2014). Crack/cocaine users show more family problems than other substance users. Clinics, 69 (7). doi: $10.6061 /$ clinics/2014(07)10

Oser, C., Leukefeld, C., Staton-Tindall, M., Duvall, J., Garrity, T., Stoops, W., ... Booth, B. (2011). Criminality among rural stimulant users in the united states. Crime \& Delinquency, 57(4), 600-621. doi: 10.1177/0011128708325048

Resolução RDC n. 29, de 30 de junho de 2011 (2011). Dispõe sobre os requisitos de segurança sanitária para o funcionamento de instituições que prestem serviços de atenção a pessoas com transtornos decorrentes do uso, abuso ou dependência de substâncias psicoativas. Recuperado de http:// bvsms.saude.gov.br/bvs/saudelegis/anvisa/2011/ res0029_30_06_2011.html

Romanini, M., \& Roso, A. (2013). Mídia, ideologia e cocaína (Crack): Produzindo "refugo humano". Psico-USF, 18(3), 373-382. Recuperado de http:// www.scielo.br/pdf/pusf/v18n3/a04v18n3.pdf

Sanchez, Z. V. D. M., \& Nappo, S. A. (2007). From the first drug to crack: The sequence of drugs taken in a group of users in the city of São Paulo. Substance Use and Misuse, 42(1), 177-188. Recuperado de http://www.ncbi.nlm.nih.gov/ pubmed/17366132

Santos, M. F. S., Neto, M. L. A., \& Sousa, Y. S. O. (2012). Representações sociais do crack na imprensa pernambucana. Estudos de Psicologia (Campinas), 29(3), 379-386. doi: 10.9788/TP2015.2-04

Scaduto, A. A., Barbieri, V., \& dos Santos, M. A. (2014). Comunidades terapêuticas para dependentes de substâncias psicoativas: Avaliação dos resultados do tratamento. Revista Psicologia-Teoria e Prática, 16(2). Recuperado de http://pepsic.bvsalud.org/ pdf/ptp/v16n2/14.pdf

Slade, E.P., Stuart, E.A., Salkever, D.S., Karakus, M., Green, K.M., \& Ialongo, N. (2008). Impacts of age of onset of substance use disorders on risk of adult incarceration among disadvantaged urban youth: A propensity score matching approach. Drug and Alcohol Dependence, 95(1-2), 1-13. doi: 10.1016/j.drugalcdep.2007.11.019

Vaughn, M. G., Fu, Q., Perron, B. E., Bohnert, A. S. B., \& Howard, M. O. (2010). Is crack cocaine use associated with greater violence than powdered cocaine use? Results from a National Sample. The American Journal of Drug and Alcohol Abuse, 36, 181186. doi: 10.3109/00952990.2010.491877

Werb, D., Kerr, T., Li, K., Montaner, J., \& Wood, E. (2008). Risks surrounding drug trade involvement among street-involved youth. The American Journal of Drug and Alcohol Abuse, 34, 810-820. doi: 10.1080/00952990802491589

Recebido em: 20/04/2015

Reformulado em: 08/07/2015 Aprovado em: 13/07/2015 
Nota das autoras:

Apoio financeiro: Fundação de Amparo à Pesquisa do Estado de Minas Gerais (FAPEMIG) - Demanda Universal (Processo n ${ }^{\circ}$ CHE - APQ-00698-12). Universidade Federal de Juiz de Fora - Edital de Iniciação Científica XXV BIC/ UFJF-2012/2013.

Sobre as autoras:

Bianca Aparecida Ribeiro Singulane é psicóloga e mestranda do Programa de Pós-Graduação em Psicologia da UFJF e integrante do grupo de pesquisa CREPEIA

E-mail: bianca.singulane@gmail.com

Nayara Baptista Silva cursa a graduação em Psicologia pela UFJF, é bolsista de iniciação científica e integrante do grupo de pesquisa CREPEIA

E-mail: nayara.baptistas@gmail.com

Laisa Marcorela Andreoli Sartes é doutora em Ciências pelo departamento de Psicobiologia da UNIFESP, professora do departamento de Psicologia e do programa de pós-graduação em Psicologia da UFJF e vice-coordenadora do Centro de Referência em Pesquisa, Intervenção e Avaliação em Álcool e Outras Drogas (CREPEIA).

E-mail: laisa.sartes@ufjf.edu.br

Contato com as autoras:

Laisa Marcorela Andreoli Sartes

Universidade Federal de Juiz de Fora

Instituto de Ciências Humanas - Departamento de Psicologia.

Rua José Lourenço Kelmer, s/n - Campus Universitário, Bairro São Pedro

CEP: 36036-900

Juiz de Fora-MG, Brasil

Psico-USF, Bragança Paulista, v. 21, n. 2, p. 395-407, mai./ago. 2016 